\title{
Randomised controlled trial of cognitive behavioural therapy in COPD
}

\author{
Karen Heslop-Marshall ${ }^{1,2}$, Christine Baker $^{3}$, Debbie Carrick-Sen ${ }^{4,5}$, \\ Julia Newton ${ }^{2}$, Carlos Echevarria ${ }^{1,2}$, Chris Stenton ${ }^{1}$, Michelle Jambon ${ }^{6}$, \\ Joanne Gray ${ }^{7}$, Kim Pearce $^{2}$, Graham Burns ${ }^{1}$ and Anthony De Soyza $\mathbb{1}^{2}$
}

Affiliations: ${ }^{1}$ Chest Clinic, Newcastle upon Tyne Hospitals NHS Foundation Trust, RVI Hospital, Newcastle upon Tyne, UK. ${ }^{2}$ Institute of Cellular Medicine, Medical School, Newcastle University, Newcastle upon Tyne, UK. ${ }^{3}$ Dept of Clinical Health Psychology, Newcastle upon Tyne Hospitals NHS Foundation Trust, RVI Hospital, Newcastle upon Tyne, UK. ${ }^{4}$ Nursing Dept, School of Medical and Dental Sciences, University of Birmingham, Birmingham, UK. ${ }^{5}$ Heart of England NHS Trust, Birmingham, UK. ${ }^{6}$ Community Chest Team, Newcastle Upon Tyne Hospitals NHS Foundation Trust, RVI Hospital, Newcastle upon Tyne, UK. ${ }^{7}$ Faculty of Health and Life Science, Northumbria University, Newcastle upon Tyne, UK.

Correspondence: Karen Heslop-Marshall, Chest Clinic, RVI Hospital, Queen Victoria Road, NE1 4LP, UK. E-mail: karen.marshall1ancl.ac.uk

ABSTRACT Anxiety is an important comorbidity in chronic obstructive pulmonary disease (COPD). We investigated if cognitive behavioural therapy (CBT), delivered by respiratory nurses, reduced symptoms of anxiety and was cost-effective.

Patients with COPD and anxiety were randomised to CBT or self-help leaflets. Anxiety, depression and quality of life were measured at baseline, 3, 6 and 12 months. A cost-effectiveness analysis was conducted from a National Health Service hospital perspective and quality-adjusted life-years estimated using the EuroQol-5D questionnaire.

In total, 279 patients were recruited. Group mean change from baseline to 3 months in the Hospital Anxiety and Depression Anxiety Subscale was 3.4 (95\% CI 2.62-4.17, p<0.001) for the CBT group and 1.88 (95\% CI 1.19-2.55, p<0.001) in the leaflet group. The CBT group was superior to leaflets at 3 months (mean difference in the Hospital Anxiety and Depression Anxiety Subscale was 1.52, 95\% CI 0.49-2.54, $\mathrm{p}=0.003$ ). Importantly, the CBT intervention was more cost-effective than leaflets at 12 months, significantly lowering hospital admissions and attendance at emergency departments.

CBT delivered by respiratory nurses is a clinically and cost-effective treatment for anxiety in patients with COPD relative to self-help leaflets.

@ERSpublications

Cognitive behavioural therapy (CBT) delivered by respiratory nurses is clinically effective and cost-effective for patients with chronic obstructive pulmonary disease and symptoms of anxiety http://ow.ly/o41o30msBNy

Cite this article as: Heslop-Marshall K, Baker C, Carrick-Sen D, et al. Randomised controlled trial of cognitive behavioural therapy in COPD. ERJ Open Res 2018; 4: 00094-2018 [https://doi.org/ 10.1183/23120541.00094-2018].

This article has supplementary material available from openres.ersjournals.com

This study is registered at www.isrctn.com with identifier number ISCRTN 55206395. The authors are happy to share the study protocol, statistical analysis plan, informed consent form, patient information leaflet, data collection form and anonymised data with researchers who provide a methodologically sound proposal. The data will be available for 5 years after the article is published.

Received: June 172018 | Accepted after revision: Oct 122018

Copyright $\odot$ ERS 2018. This article is open access and distributed under the terms of the Creative Commons Attribution Non-Commercial Licence 4.0. 


\section{Introduction}

Chronic obstructive pulmonary disease (COPD) is one of the most important chronic diseases affecting over 210 million people worldwide [1]. Anxiety and depression are major comorbidities in COPD associated with high morbidity, disability, healthcare expenditure and mortality $[2,3]$. The under diagnosis and under treatment of anxiety and depression in COPD is common and can adversely affect patient outcomes [4-6]. Anxiety and depression have a significant impact on physical functioning, breathlessness, quality of life, exacerbation rates, use of healthcare resources, length of hospital stay, readmission rates and mortality [2-4, 7-11]. Patients with psychological difficulties are less able to manage symptoms [12] and are less likely to be physically active [4] or attend pulmonary rehabilitation, an evidence-based intervention, which is a key strategy for people with chronic respiratory conditions [13].

Guidelines for the treatment of anxiety and depression recommend psychological treatment (cognitive behavioural therapy (CBT), counselling and self-help approaches) and pharmacological treatment, or a combination of both $[14,15]$. CBT is a "talking" treatment, which explores the link between a patient's situation, physical symptoms, thoughts, emotions and behaviour.

Traditionally, treatment for anxiety and depression is provided by mental health professionals, but this may not encompass the needs of COPD patients where complex physical health problems and mental health issues are interrelated. There are also barriers to accessing mental health support including perceived stigma and unwillingness to engage with mental health services, lack of prioritisation compared with physical healthcare [16], increasing demand and limited resources [16]. While CBT is an evidence-based intervention for anxiety and depression generally, the effectiveness of CBT in the context of COPD is unclear $[17,18]$. The need for high-quality research with sufficient power to test the potential benefit of CBT in COPD has been recommended [17-19]. We identified anxiety as the most appropriate primary outcome due to the frightening nature of breathlessness and the link with increased use of healthcare resources [20]. We designed a pragmatic randomised controlled trial (RCT) with sufficient power to address our hypothesis that one-to-one CBT sessions, delivered by respiratory nurses, could lead to a reduction in anxiety symptoms as measured by the Hospital Anxiety and Depression Scale (HADS) Anxiety Subscale [21], and could be a cost-effective intervention.

\section{Materials and methods}

Study design

A pragmatic RCT was conducted according to ethical principles outlined in the Declaration of Helsinki 2008 and following the methods set out in a published protocol (ISCRTN 55206395) [22]. The study was approved by the National Research Ethics Service (11/NE/0025).

\section{Patients}

Study accessibility was maximised with pragmatic inclusion and exclusion criteria to mirror real life clinical settings (table 1 ).

\section{Study interventions}

A remote computerised program and concealed sequence generation was used to randomise patients to an active control of self-help leaflets addressing anxiety management $[24,25]$ or a brief CBT intervention plus self-help leaflets [24, 25]. All patients received standard medical care (spirometry, medical review, pharmacological treatment (e.g. respiratory medication, antidepressants and anxiolytics)) and pulmonary rehabilitation if eligible.

\section{TABLE 1 Inclusion and exclusion criteria}

Inclusion criteria
A confirmed diagnosis of COPD (FEV $1 / F V C$
ratio $<70 \%[5]$
All levels of COPD disease severity
(mild-very severe)
HADS-Anxiety Subscale score of $\geqslant 8$
Consent to $2-6$ sessions of CBT [23]

COPD: chronic obstructive pulmonary disease; FEV1: forced expiratory volume in $1 \mathrm{~s}$; FVC: forced vital capacity; HADS: Hospital Anxiety and Depression Scale; CBT: cognitive behavioural therapy.

\section{Exclusion criteria}

A HADS-Anxiety Subscale score of $<8$ Known psychiatric history such as psychosis

Patients currently receiving psychological talking therapy including CBT treatment

Patients with cognitive impairment (e.g. dementia) Patients involved in any other interventional clinical trial 
The brief CBT intervention was developed and refined before the study commenced (the Lung Manual) [26]. Between two and six fortnightly CBT sessions were undertaken, which is recommended for a brief intervention [23]. Sessions were conducted either in clinic or at home and lasted $\sim 30 \mathrm{~min}$. The number of sessions suggested and timescale for these was then co-developed with the patients depending on the patients' individual response to treatment to manage symptoms based on their subjective feedback, HADS questionnaire results and patient preference. The respiratory nurses used cognitive and behavioural techniques to reduce symptoms of anxiety. Key components of the treatment are summarised in supplementary table S1.

\section{Nurse training}

Four respiratory nurses with varying clinical experience post-qualification (1-30 years) were recruited to deliver the intervention. Two nurses had completed a post-graduate diploma in CBT. Two nurses completed 3 days CBT training (table 2).

The nurses attended the first 2 days training then returned to clinical practice for 6 weeks to consolidate their skills with patients within their clinical setting. On day three the nurses presented a case study to demonstrate learning and application of CBT skills. The nurses and a patient carer from the Trial Steering Committee attended a half day training on the Lung Manual.

To formally assess CBT skills and treatment fidelity, random CBT sessions were video-recorded and rated using the Cognitive First Aid rating scale (CFARS) 10-item scale [27]. The CFARS scores range from zero (poor skills and competency) to six (high level of skill and competency). Scoring four or above is considered an acceptable level of skills and expertise [15]. The nurses received monthly clinical supervision (with K.H.M.) who developed the intervention. This is essential to gain further skill and maintain confidence [27].

\section{Outcome measurements}

The primary outcome for anxiety was the change in the group mean HADS-Anxiety Subscale at 3 months from baseline. The HADS is a brief self-completed questionnaire, validated in COPD [28, 29]. Scores of 8-10 indicates mild symptoms, 11-14 moderate and 15-21 severe [28]. The minimal clinically important difference (MCID) of HADS-Anxiety is 1.32 and 1.40 for HADS-Depression [29]. A difference of the HADS (anxiety or depression) of 1.5 is recommended in COPD patients corresponding to a change from baseline of $\sim 20 \%$ [29].

Secondary outcomes were the change in group mean HADS-Anxiety Subscale at 6 and 12 months from baseline and group mean change in the HADS-Depression Subscale, COPD Assessment Test (CAT) [30] and EuroQol-5D (EQ-5D) 3L [31] at 3,6 and 12 months from baseline. The CAT is a disease specific health status questionnaire containing eight items and has been validated in COPD [30]. A change of two or more units suggests a clinically significant difference in COPD health status [32]. The EQ-5D 3L is a generic quality of life questionnaire, which is commonly used for cost-effectiveness analysis of interventions using quality-adjusted life-years (QALYs). The QALY incorporates the impact on both the quantity and quality of life. The EQ-5D $3 \mathrm{~L}$ is a reliable and valid tool for COPD patients [33]. Staff collecting the outcome data were blinded to the intervention.

\section{TABLE 2 Content of cognitive behavioural therapy (CBT) nurse training}
Day 1
Day 2

Course aims

Basic introduction to CBT

Using CBT in physical health setting

Anxiety

Panic

Depression

Screening for anxiety and depression

Risk management (suicide assessment)

Formulation of patients difficulties

Socratic questioning and guided discovery

Unhelpful thinking

Practical session
Revision

Feedback from homework

Goal setting

Cognitive techniques

Behavioural techniques

Case study

Practical session (theory into practice)

Summary

Homework
Day 3

Feedback from homework Revision

Case presentations

Supervision

Course evaluation 


\section{Statistical analysis}

The sample size calculation was based on a two-sample t-test with a pooled standard deviation of four for the HADS-Anxiety Subscale based on a local sample of patients with COPD. Complete outcome data on 112 patients per group was required to detect a standardised difference of 0.375 (equivalent to a $20 \%$ improvement in HADS-Anxiety score) with $80 \%$ power at the $95 \%$ significance level [29].

An intention-to-treat analysis was undertaken. Data were analysed for all participants, for whom an outcome was available, according to the treatment group to which they were randomised. Means and percentages were used for descriptive statistics. For the primary outcome, groups were compared using an independent t-test. A secondary analysis was performed using multiple imputation for missing data. Multiple imputation is a statistical technique for analysing incomplete data [34]. Fisher's exact test was used to compare categorical data where applicable, tests were two-tailed and significance was set at a level of $\mathrm{p}<0.05$.

\section{Results}

From June 2011 to October 2014, 1518 COPD outpatients were screened. We found 898 (59\%) of patients had a HADS-Anxiety Subscale of eight or more. An outline of patient flow can be found in figure 1 . Baseline characteristics are presented in table 3. The mean age for all patients was 66 years (range 41-88 years). All patients recruited had symptoms of anxiety with a HADS-Anxiety Subscale of eight or above. The mean HADS-Anxiety Subscale was 12.3 for the CBT group and 12.0 for the self-help leaflet group at baseline, suggesting moderate anxiety symptoms [11]. $64 \%$ of patients reported moderate to severe symptoms of anxiety. Coexisting symptoms of depression were reported in 186 (67\%): 94 in the CBT group (68\%) and $92(66 \%)$ in the self-help leaflet group at baseline. There appeared to be no correlation between HADS-Anxiety or Depression scores at baseline and severity of lung function based on forced expiratory volume in $1 \mathrm{~s}(\mathrm{FEV} 1) \%$ predicted.

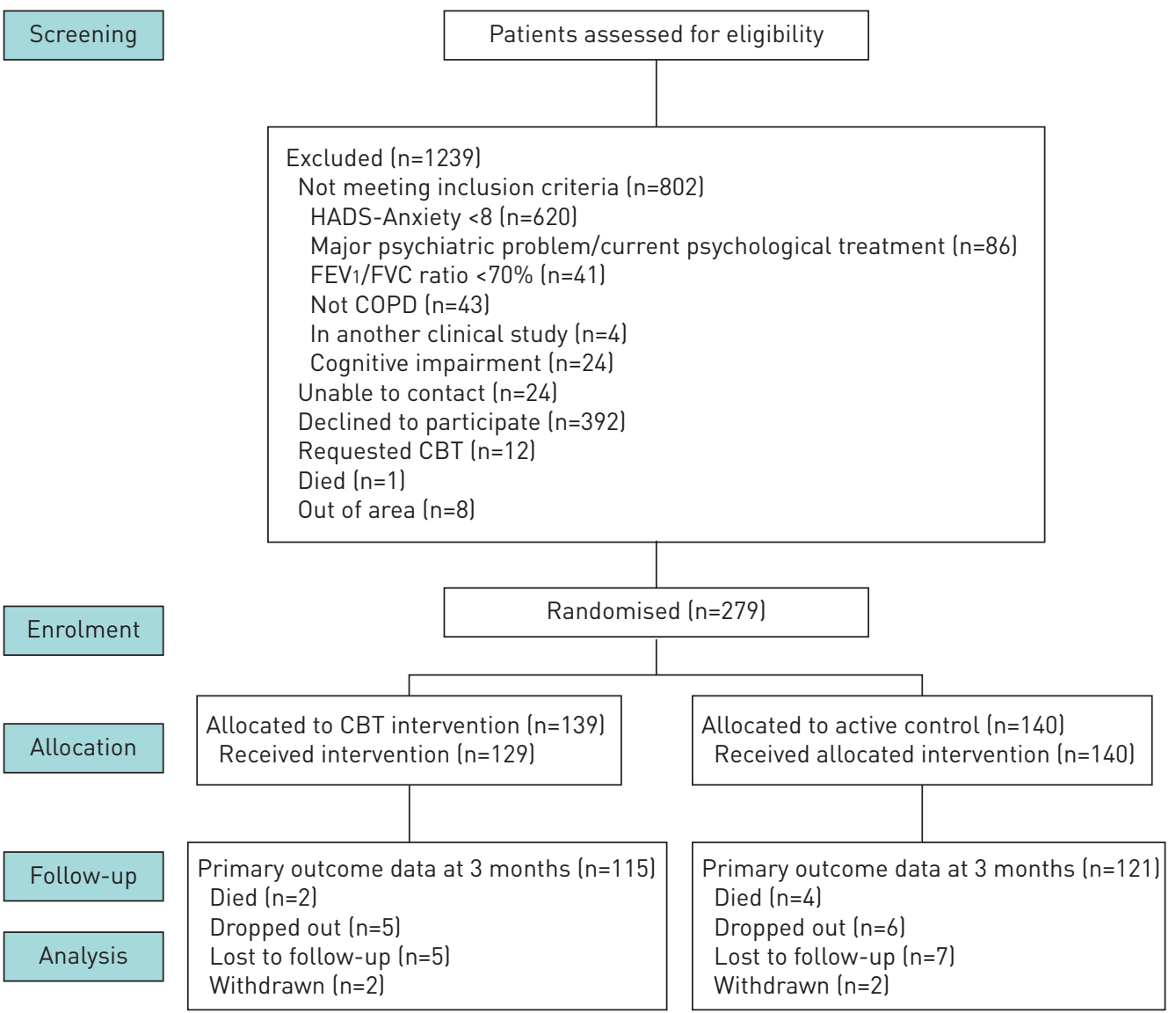

FIGURE 1 Patient flow diagram. HADS: Hospital Anxiety and Depression Scale; FEV1: forced expiratory volume in $1 \mathrm{~s}$; FVC: forced vital capacity; COPD: chronic obstructive pulmonary disease; CBT: cognitive behavioural therapy. 


\begin{tabular}{|c|c|c|c|}
\hline & СВТ group & Leaflet group & Total patients \\
\hline Subjects $n$ & 139 & 140 & 279 \\
\hline Mean士sD age years & $66 \pm 10.2$ & $67 \pm 9.6$ & $66.5 \pm 9.9$ \\
\hline Males/females & $61(44) / 78(56)$ & $67(48) / 73(52)$ & $128(46) / 151(54)$ \\
\hline Ethnic group, Caucasian & $139(100)$ & $140(100)$ & $279(100)$ \\
\hline Mean \pm SD HADS-Anxiety Subscale & $12.3 \pm 3.19$ & $12.0 \pm 2.94$ & $12.2 \pm 3.06$ \\
\hline HADS-Anxiety Subscale 8-10 & 51 (37) & 49 (35) & $100(36)$ \\
\hline HADS-Anxiety Subscale 11-14 & 52 (37) & $63(45)$ & $115(41)$ \\
\hline HADS-Anxiety Subscale $15-21$ & $36(26)$ & $28(20)$ & 64 (23) \\
\hline Mean $\pm s D$ HADS-Depression Subscale & $9.2 \pm 4.0$ & $8.8 \pm 3.7$ & $9.0 \pm 3.85$ \\
\hline \multicolumn{4}{|c|}{ Severity of airflow obstruction based on GOLD criteria } \\
\hline Mild & $16(11)$ & $13(9)$ & $29(10)$ \\
\hline Moderate & $44(32)$ & 47 (34) & $91(33)$ \\
\hline Severe & $50(36)$ & $49(35)$ & $99(35)$ \\
\hline Very Severe & $29(21)$ & $31(22)$ & $60(22)$ \\
\hline \multicolumn{4}{|l|}{ MRC Breathlessness score } \\
\hline $0-2$ & $8(6)$ & $12(9)$ & $20(7)$ \\
\hline 3 & $25(18)$ & $19(14)$ & $44(16)$ \\
\hline 4 & $39(28)$ & $44(31)$ & $83(30)$ \\
\hline 5 & $67(48)$ & $65(46)$ & $132(47)$ \\
\hline Current smoker & $39(28)$ & 40 (29) & $79(28)$ \\
\hline 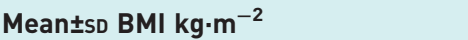 & $26 \pm 6.4$ & $27 \pm 6.0$ & $26.5 \pm 6.22$ \\
\hline No educational qualifications & $100(72)$ & $103(74)$ & $203(73)$ \\
\hline
\end{tabular}

In the CBT group, 10 patients did not receive the allocated treatment due to dropping out or were found at pretreatment assessment to be ineligible (due to undiagnosed cognitive impairment or undisclosed formal psychiatric involvement at the time of consent).

Self-reported data was collected at baseline on relevant pharmacological therapy (see supplementary table S2). In total, 52 (37\%) patients had been prescribed antidepressants compared with 47 (34\%) from the leaflet group. Data on pulmonary rehabilitation was collected at baseline and 12 months. 46 patients had completed pulmonary rehabilitation in the CBT group at baseline and this increased to 64 patients at 12 months. In the leaflet group 50 patients had completed pulmonary rehabilitation at baseline and this increased to 62 at 12 months.

\section{Anxiety}

In total, 236 patients completed the primary end-point of 3 months ( $85 \%$ completion rate). Complete data were available for 115 patients in the CBT arm and 121 in the leaflet arm. Group mean data for HADS-Anxiety can be seen in table 4 [29].

The average number of CBT sessions delivered was four, which falls within the recommendations for a brief intervention [23]. There was a statistically significant improvement in mean HADS-Anxiety scores in

TABLE 4 Group mean Hospital Anxiety and Depression (HADS)-Anxiety Subscale at each time point

Group mean士sD HADS-Anxiety Subscores

\begin{tabular}{lcccc} 
& Baseline & $\mathbf{3}$ months & $\mathbf{6}$ months & $\mathbf{1 2}$ months \\
\hline Cognitive behavioural therapy & $12.3 \pm 3.19$ & $8.8 \pm 4.49$ & $8.6 \pm 4.02$ & $8.7 \pm 4.06$ \\
$(\mathrm{n}=139)$ & $(\mathrm{n}=115)$ & $(\mathrm{n}=93)$ & $\begin{array}{c}(\mathrm{n}=93) \\
\text { Leaflet }\end{array}$ \\
\cline { 2 - 5 } & $\begin{array}{c}12.0 \pm 2.94 \\
(\mathrm{n}=140)\end{array}$ & $\begin{array}{c}10.0 \pm 4.42 \\
(\mathrm{n}=121)\end{array}$ & $\begin{array}{c}9.7 \pm 4.31 \\
(\mathrm{n}=99)\end{array}$ & $\begin{array}{c}10.2 \pm 4.33 \\
(\mathrm{n}=79)\end{array}$ \\
\end{tabular}


the CBT group compared to the self-help leaflet group at 3 months (table 5). For the HADS-Anxiety Subscale change score, the difference between the two treatment groups is significant at the table wide 0.05 level after applying the sequential Bonferroni method [35].

A secondary outcome was a reduction in symptoms of depression. The mean HADS-Depression Subscale for the two groups were compared at 3,6 and 12 months. The CBT arm was statistically significantly better than the leaflet arm at 3 and 6 months, but this did not reach the MCID (see supplementary table S3).

\section{CBT competency and fidelity}

During the study 22 CBT sessions were video recorded and assessed to evaluate adherence to the Lung Manual and competency delivering the CBT. The validated 10-item CFARS was used [27]. The scoring system for the items ranges from zero (poor skills and competency) to six (high level of skill and competency). Scoring four or above is considered an acceptable level of skills and expertise. Average competency scores for CFARS rating scale are summarised in supplementary table S4. The nurses scored highly on focus/structure of the CBT, therapeutic relationship and providing feedback to patients.

\section{Health economic analysis}

A health economic analysis from a National Health Service (NHS) perspective (hospital setting) was conducted alongside the RCT to evaluate the cost-effectiveness of the CBT intervention compared to self-help leaflets [36]. The EQ-5D 3L scores collected at baseline, 3-, 6- and 12-months post-randomisation were converted into QALYs using the area under the curve method with adjustment for baseline utility scores [37]. The costs and benefits were not discounted since the timescale of the trial was 12 months.

NHS costs (including intervention costs and costs of respiratory-related hospital admissions and attendance at emergency departments) were compared with QALYs using established methods [38]. All resource use was costed at 2015/2016 prices in GBP. The costs of the CBT intervention comprised of staff time (including the costs of training to deliver the intervention in terms of staff time, delivering the intervention and ongoing supervision). Both groups received the self-help leaflet, so we assumed this cost was equal. Unit cost data were obtained from a combination of local costings and national databases [39, 40].

Based on the EQ-5D missing quality of life observations were assigned using multiple imputation by chained equation methods using variables related to quality of life including age, gender, marital status and education [36]. Results are presented using an incremental cost-effectiveness ratio (ICER). To gain an understanding of the uncertainty surrounding the ICER, a cost-effectiveness acceptability curve was estimated using a nonparametric bootstrap method on the basis of 5000 replications. This shows the probability of each comparator being cost-effective given a particular level of the decision maker's willingness to pay for an additional QALY [36].

The patients randomised to CBT had fewer hospital admissions and fewer emergency department attendances ( 0.6 versus 1.01 and 0.37 versus 1.01, respectively). The CBT intervention cost GBP 175 per patient (approximately EUR 200), which resulted from extra initial costs being incurred (including the costs of training to deliver the intervention in terms of staff time, delivering the intervention and ongoing supervision) relative to the self-help leaflets (95\% CI 165-185, p=0.001) (table 6). However, costs were offset by lower subsequent costs of respiratory hospital admissions with an average mean difference of GBP 1089 (95\% CI -2370-227) (approximately EUR 1247) saved per patient and GBP 63 (95\% CI $-121--7$ ) (approximately EUR 72) lower for emergency department attendances. The results for EQ-5D 3L show that both groups experienced an increase in QALYs; however, the CBT arm gained more QALYs and

TABLE 5 Differences in mean anxiety from baseline and between groups

Mean improvement from baseline

CBT group

$3.40(n=115)$

$3.42(n=101)$

$3.35(\mathrm{n}=93)$
Leaflet group

$1.88(n=121)$

$2.36(n=99)$

$1.89(n=79)$
Difference in mean improvement ( $95 \% \mathrm{Cl}, \mathrm{p}$-value)

$1.52^{\#}(0.49-2.54, p=0.003)$

$1.05(-0.04-2.14, p=0.05)$

$1.43(0.28-2.66, p=0.016)$

CBT: cognitive behavioural therapy; HADS: Hospital Anxiety and Depression Scale. ${ }^{\text {: }}$ the mean difference is higher than the HADS-Anxiety Score minimal clinically important difference of 1.50 [17]. 
was the most effective with an average mean difference of 0.079 ( $95 \%$ CI $0.023-0.140, p=0.008$ ). Thus, the CBT intervention was less expensive and more effective than self-help leaflets alone.

The results suggest that the intervention is a dominant strategy relative to self-help leaflets. To gain an understanding of the uncertainty surrounding the dominance of CBT, a cost-effectiveness acceptability curve (CEAC) was estimated using a nonparametric bootstrap method based on 5000 replications [36]. The results of the parametric CEAC and the cost-effectiveness plane are presented in figure 2. At all thresholds for the willingness to pay for a QALY, CBT has a higher probability of being cost-effective compared with self-help leaflets (table 7). For values over GBP 5000 (approximately EUR 5727), the probability that the intervention is cost-effective is $100 \%$.

Overall, the CBT intervention was less expensive (driven by the reduction in resource use with lower hospital admissions and a statistically significant reduction in emergency department attendances) than self-help leaflets. These results suggest there is minimal uncertainty that CBT is more effective and less expensive than self-help leaflets.

\section{Discussion}

In the largest study to date, we hypothesised that one-to-one CBT sessions delivered by respiratory nurses could lead to a reduction in anxiety as documented by the HADS-Anxiety Subscale [21] and could be cost-effective across a range of COPD severities. We found a significant number of patients screened (59\%) had symptoms of anxiety. This prevalence is higher than an earlier systematic review and meta-analysis that identified anxiety symptoms in $46 \%$ of patients [3]. Over half of patients also had comorbid symptoms of depression.

The results indicate CBT delivered by respiratory nurses was clinically effective in patients with COPD and comorbid symptoms of anxiety. The CBT intervention was both statistically and clinically superior to self-help leaflets in the primary outcome measured at 3 months with a difference of 1.52 on the HADS-Anxiety Subscale at the primary end-point [29].

The CBT intervention was also cost-effective. While the CBT intervention incurred initial costs for training and supervision, these costs were offset by lower subsequent costs for respiratory admissions and emergency department attendances over 12 months. The results of the EQ-5D 3L analysis also show that while both groups experienced an increase in QALYs, the CBT arm gained more and was the most effective. We noted that there was no correlation between lung function (FEV1 \% predicted) and HADS-Anxiety Subscale, which may suggest patients with milder COPD treated within primary care services may benefit from this clinically and cost-effective treatment. Potential mechanisms underlying reduced healthcare utilisation include better self-management of frightening physical symptoms such as breathlessness, amplified by anxiety. As patients did not have to have symptoms of depression at baseline, the benefit of CBT cannot be concluded from this study. We believe the HADS-Depression data are interesting and support an effect of CBT on HADS-Depression, but further research is needed.

\section{Strengths and limitations}

The strengths of this pragmatic multicentre study are that it was adequately powered and only included patients with validated objective measurements of anxiety symptoms at baseline. We also included all levels of COPD severity to improve generalisability. Further strengths are that we used processes to minimise bias from randomisation, data collection and data analysis. This addresses the limitations of previous studies [17]. The results were confirmed using multiple imputation for missing data suggesting bias due to dropouts was unlikely.

Complex behaviour change interventions are often poorly described when reported, impairing the ability to replicate the research [41]. A detailed manual and training programme were developed to allow its use to be replicated.

A comprehensive health economic evaluation was conducted according to best practice methods. Our results show the CBT intervention was effective in gaining QALYs, within the National Institute for Health and Care Excellence (NICE) threshold, of GBP 30 000/EUR 34000, often applied in the UK to determine if an intervention is acceptable. At this level, the CBT intervention would be a preferable intervention and cost-effective compared to self-help leaflets [42].

Several limitations of this study are acknowledged. Caution should be used when interpreting the results of HADS questionnaire. The HADS questionnaire has been shown to be a reliable measure of emotional distress but not in predicting probable presence or "caseness" of anxiety. We cannot conclude if there is a specific HADS threshold where the intervention is more or less likely to be effective as we were not powered for subgroup analysis. Although the majority of COPD costs relate to hospital admissions, we do 
TABLE 6 Costs and QALYS of cognitive behavioural therapy (CBT) intervention and self-help leaflets

\begin{tabular}{|c|c|c|c|}
\hline & $\begin{array}{l}\text { CBT intervention } \\
\text { mean }(95 \% \mathrm{CI})\end{array}$ & $\begin{array}{c}\text { Leaflets } \\
\text { mean }(95 \% \mathrm{CI})\end{array}$ & $\begin{array}{c}\text { Bootstrapped mean difference } \\
\qquad(95 \% \mathrm{Cl}, \mathrm{p} \text {-value })\end{array}$ \\
\hline Intervention costs GBP & 175 (165-185) & & $175(165-185, p=0.001)$ \\
\hline Costs of respiratory admissions GBP & $1601(936-2267)$ & $2689(1632-3746)$ & $-1089(-2370-227, p=0.076)$ \\
\hline Number of ED attendances & $0.37(0.16-0.59)$ & $0.81(0.48-1.14)$ & \\
\hline Costs of ED attendances GBP & $55(23-86)$ & $118(70-166)$ & $-63(-121--7, p=0.036)$ \\
\hline
\end{tabular}

not have primary care attendance data. This was beyond the scope of our data collection resources. Wider societal cost analyses, including primary and community care costs, to observe any cost shifting between sectors would have been beneficial in assessing the potential wider benefits of the intervention.

There are challenges to implementing CBT for psychological morbidity associated with physical illnesses including cost, the availability of trained CBT therapists and patient reluctance to engage in psychological treatments [27]. In our study, $392(26 \%)$ eligible patients declined to take part in the trial. While this is generally in line with clinical trials engagement, the rates of engagement with CBT outside of a clinical trial are unknown. Screening for comorbid anxiety symptoms in the clinic and having a nurse skilled in both respiratory and CBT management may remove stigma or other barriers to CBT, improve engagement and allow more holistic care.

During the treatment phase, it was not possible to blind participants or the nursing staff delivering the intervention. To reduce bias, we had an independent and blinded team collecting follow-up data. We did not include a placebo group; having identified anxiety symptoms as an issue, we felt it was unethical to offer no treatment. Our control arm was self-help leaflets.

Our pragmatic study shows that tailored CBT approaches can be developed for COPD patients, helping to manage difficult symptoms of anxiety, which are intrinsically linked with breathlessness [43]. This is the

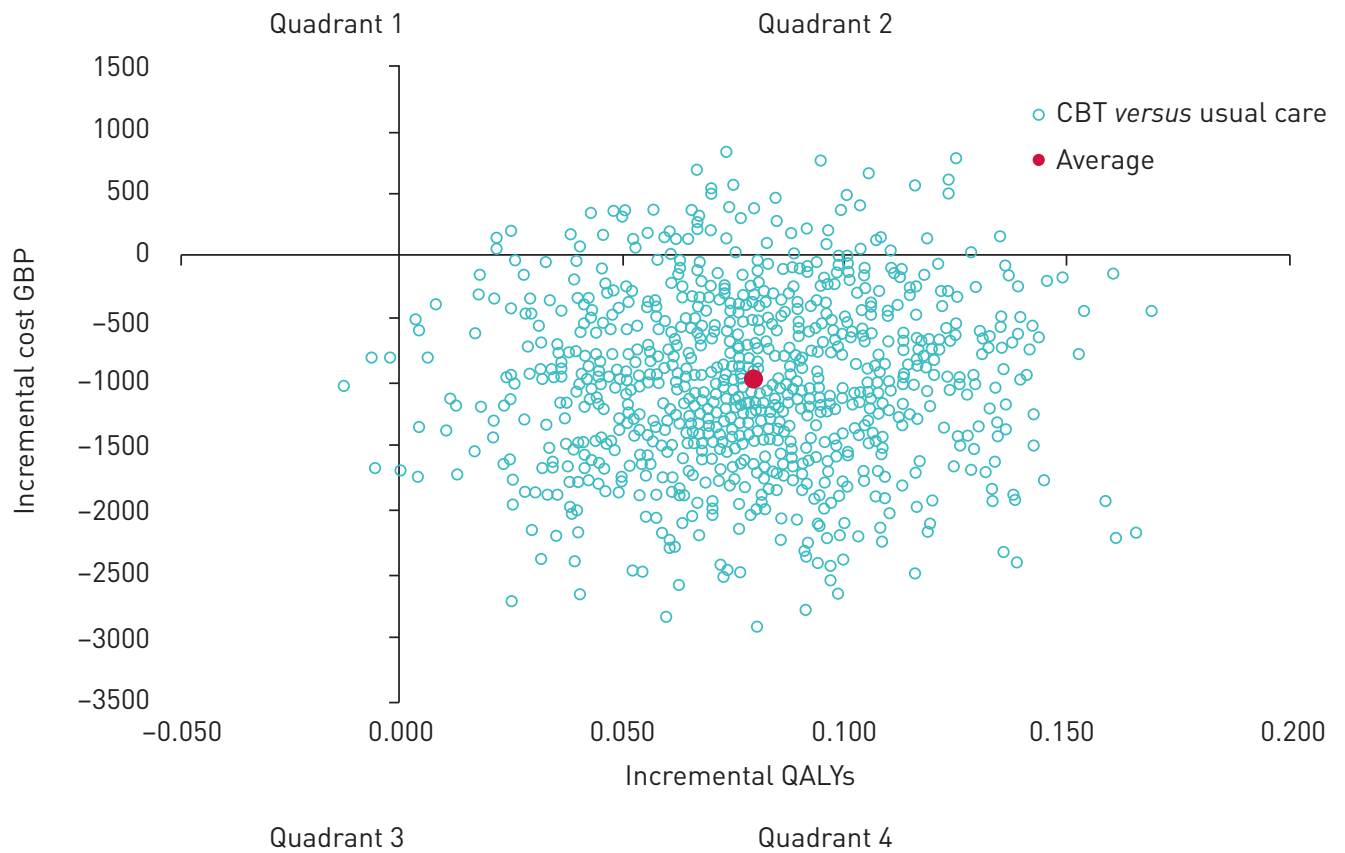

FIGURE 2 Cost-effectiveness plane. Quadrant 1: more costly/less effective; quadrant 2: more costly/more effective; quadrant 3: less costly/less effective; quadrant 4: less costly/more effective; CBT: cognitive behavioural therapy; QALYs: quality-adjusted life-years. 


\begin{tabular}{|c|c|c|c|c|c|}
\hline & \multicolumn{5}{|c|}{$\begin{array}{l}\text { Probability of each management strategy being considered } \\
\text { cost-effective at different threshold values for society's willingness } \\
\text { to pay for a QALY }\end{array}$} \\
\hline & GBP 0 & GBP 5000 & GBP 10000 & GBP 20000 & GBP 30000 \\
\hline Cognitive behavioural therapy & 0.94 & 0.98 & 1.00 & 1.00 & 1.00 \\
\hline Leaflets & 0.06 & 0.02 & 0.00 & 0.00 & 0.00 \\
\hline
\end{tabular}

largest trial of CBT in COPD, to date, and the first study to demonstrate that respiratory nurses can be trained to undertake and effectively deliver a cost-effective brief CBT intervention to reduce symptoms of anxiety in COPD patients. Training respiratory staff can lead to better recognition and treatment of breathlessness and anxiety symptoms, in patients who otherwise may not have engaged with mental health services. With greater availability of CBT trained respiratory nurses, routine screening and treatment for symptoms of anxiety in patients with COPD is feasible and should be incorporated into clinical care pathways, addressing the current lack of resources to address mental health needs [16]. Finally, further studies are needed to identify how to incorporate CBT into routine clinical care and identify if the intervention can be successfully replicated for those treated in primary care.

\section{Conclusion}

This study shows that a brief CBT intervention, uniquely delivered by respiratory nurses, was clinically effective at reducing anxiety symptoms and was cost-effective. Costs were reduced by lower resource use with lower hospital admissions and a reduction in emergency department attendances. Respiratory nurses with dual respiratory and CBT skills can address common comorbid symptoms of anxiety that were previously under treated. CBT should be incorporated into routine clinical care pathways.

Acknowledgements: We are grateful to Nick Steen, Medical Statistician (Institute of Health and Society, Newcastle University, Newcastle upon Tyne, UK), for his advice during study development.

We are grateful to patient advisors, colleagues for referring participants, research nurse Susan Leach from the NIHR-Mental Health Research Network (NIHR CNR, Leeds, U) for data collection and Gill Satterley, CBT Independent Consultant (Newcastle upon Tyne, UK) who provided CBT expertise and assessed the video sessions.

Conflict of interest: D. Carrick-Sen has nothing to disclose. C. Baker reports grants from National Institute for Health Research (NIHR)/Health Education England (support for part-time PhD fellowship for K. Heslop-Marshall), non-financial support from University of Newcastle upon Tyne (advice for Nick Steen, Medical Statistician, Newcastle University), during the conduct of the study. J. Gray has nothing to disclose. J. Newton has nothing to disclose. C. Stenton has nothing to disclose. M. Jambon has nothing to disclose. K. Pearce has nothing to disclose K. Heslop-Marshall reports grants from National Institute for Health Research (UK), during the conduct of the study; and other from Pivotal Health Education Ltd, outside the submitted work. (This research was completed in February 2016. In 2017, K. Heslop-Marshall set up a company to provide cognitive behavioural therapy (CBT) training for healthcare professionals who would like to learn foundation level skills in CBT to work with patients with long-term conditions who have psychological distress. This training will not just benefit professionals working in the respiratory setting. I did this to enhance the psychological skills and pass on my knowledge and expertise in CBT. This did not influence the research submitted in any way.) G. Burns reports personal fees from BI, Chiesi and AZ (for advice on inhalers, not directly relevant to this study), personal fees from Teva (for three educational (non-promotional) talks on COPD, not related to this study), personal fees from Chiesi (for three educational (non-promotional) talks on COPD, not related to this study), personal fees from Pfizer and AZ (for educational (non-promotional) talks on COPD, not related to this study), all outside the submitted work. A. De Soyza reports other from AstraZeneca (bronchiectasis interest group meeting support), non-financial support from Novartis (in kind support for analysis in bronchiectasis), non-financial support from Forest labs (bronchiectasis interest group meeting support), personal fees from Bayer (speakers'/advisory boards on bronchiectasis), personal fees and other from Novartis (advisory boards on bronchiectasis and in kind bench science support), other from Chiesi (travel bursary to attend ERS meeting), other from Almirall (travel bursary to attend ERS meeting), other from Boehringer Ingelheim (travel bursary to attend BTS meeting), personal fees from AstraZeneca (speakers'/advisory boards on COPD), grants from AstraZeneca (travel bursary to attend ATS meeting), all outside the submitted work. In addition, A. De Soyza has received medical education grant support for a UK bronchiectasis network from GSK, Gilead, Chiesi and Forest labs. A. De Soyza's employing institution receives fees for his work as Coordinating investigator in a phase III trial in Bronchiectasis sponsored by Bayer. C. Echevarria has nothing to disclose.

Support statement: This study was supported by the Department of Health, National Institute for Health Research, Research Trainees Coordinating Centre 10-023. This article presents independent research funded as part of a part-time $\mathrm{PhD}$ fellowship to K. Heslop-Marshall funded by the National Institute for Health Research (NIHR) and includes work 
funded by Health Education England. The views expressed are those of the author(s) and not necessarily those of the NHS, the NIHR or the Department of Health. Funding information for this article has been deposited with the Crossref Funder Registry.

\section{References}

1 Global Initiative for Chronic Obstructive Lung Disease. Global Strategy for the Prevention, Diagnosis and Management of COPD. 2017. www.goldcopd.org

2 Maurer J, Rebbapragada V, Borson S, et al. Anxiety and depression in COPD : current understanding, unanswered questions, and research needs. Chest 2008; 134: Suppl., 43S-56S.

3 Yohannes AM, Kaplan A, Hanania NA. Anxiety and depression in chronic obstructive pulmonary disease: recognition and management. Cleve Clin J Med 2018: S11-SS8.

4 Yohannes AM, Alexopoulos GS. Depression and anxiety in patients with COPD. Eur Respir Rev 2014; 23 345-349.

5 Pumar MI, Gray CR, Walsh JR, et al. Anxiety and depression - important psychological comorbidities of COPD J Thorac Dis 2014; 6: 1615-1631.

6 Dury R. COPD and emotional distress: not always noticed and therefore undertreated. Br J Commun Nurs 2016; 21: $138-141$.

7 Gudmundsson G, Gislason T, Janson C, et al. Risk factors for rehospitalisation in COPD: role of health status, anxiety and depression. Eur Respir J 2005; 26: 414-419.

8 Atlantis E, Fahey P, Cochrane B, et al. Birdirectional associations between clinically relevant depression or anxiety and COPD: a systematic review and meta-analysis. Chest 2013; 144: 766-777.

9 Ng TP, Niti M, Tan WC, et al. Depressive symptoms and chronic obstructive pulmonary disease: effect on mortality, hospital readmission, symptom burden, functional status and quality of life. Arch Intern Med 2007; 167: $60-67$.

10 Giardino ND, Curtis JL, Abelson JL, et al. The impact of panic disorder on interoception and dyspnea reports in chronic obstructive pulmonary disease. Biol Psychol 2010; 84: 142-146.

11 Livermore N, Dimitrice A, Sharpe L, et al. Cognitive behaviour therapy reduces dyspnoea ratings in patients with chronic obstructive pulmonary disease (COPD). Respir Physiol Neurobiol 2015; 216: 35-42.

12 Thew GR, MacCallam J, Salkovskis PM, et al. Developing and evaluating psychological provision in the acute hospital setting for patients with chronic respiratory disease. The Cognitive Behavioural Therapist 2017; 10: E5 doi: 10.1017/S1754470X17000071.

13 Bolton CE, Bevan-Smith EF, Blake JD, et al. British Thoracic Society guideline on pulmonary rehabilitation in adults. Thorax 2013; 68: Suppl., iil-ii30.

14 National Institute for Clinical Excellence. Depression in adults with a chronic physical health problem: recognition and management. Clinical guideline [CG91]. National Institute for Clinical Excellence, 2010.

15 National Institute for Health and Clinical Excellence. Generalised Anxiety Disorder and Panic Disorder (with or without agoraphobia) in Adults: Management in Primary, Secondary and Community Care. Leicester, The British Psychological Society and The Royal College of Psychiatrists, 2011.

16 BMA. Breaking down barriers - the challenge of improving mental health outcomes. London, British Medical Association, 2017.

17 Baraniak A, Sheffield D. The efficacy of psychologically based interventions to improve anxiety, depression and quality of life in COPD: a systematic review and meta-analysis. Patient Educ Couns 2011; 83: 29-36.

18 Usmani ZA, Carson KV, Heslop K, et al. Psychological therapies for the treatment of anxiety disorders in chronic obstructive pulmonary disease. Cochrane Database Syst Rev 2017; 3: CD010673.

19 Yohannes AM, Junkes-Cunha M, Smith J, et al. Management of dyspnea and anxiety in chronic obstructive pulmonary disease: a critical review. J Am Med Dir Assoc 2017; 18: 1096.e1-1096.e17.

20 Pooler A, Beech R. Examining the relationship between anxiety and depression and exacerbations of COPD which result in hospital admission: a systematic review. Int J Chron Obstruct Pulmon Dis 2014; 9: 315-330.

21 Zigmond AS, Snaith RP. The Hospital Anxiety and Depression Scale. Acta Psychiatr Scand 1983; 67: 361-370.

22 Heslop-Marshall K. Newcastle Chronic Obstructive Pulmonary Disease (COPD) cognitive behavioural therapy (CBT) Care Study: a randomised controlled trial. Newcastle, University of Newcastle, 2016.

23 Care Services Improvement Partnership. IAPT Outline Service Specification. Institute for Mental Health England. www.uea.ac.uk/documents/246046/11991919/iapt-pathfinder-outline-service-specification.pdf/9fc11891-ecc5-48e4a974-9bbc8ab0c690

24 Maunder L, Cameron L. Depression and Low Mood. https://web.ntw.nhs.uk/selfhelp/leaflets/Depression\%20and\% 20Low\%20Mood\%20A4\%202016\%20FINAL.pdf Northumberland, Tyne \& Wear Mental Health Trust, 2011.

25 Maunder L, Cameron L. Panic. A self-help guide. https://web.ntw.nhs.uk/selfhelp/leaflets/Panic\%20A4\%202016\% 20FINAL.pdf Northumberland Tyne \& Wear NHS Foundation Trust, 2011.

26 Heslop K, Foley T. Using cognitive behavioural therapy to address the psychological needs of patients with COPD. Nurs Times 2009; 105: 18-19.

27 Mannix KA, Blackburn IM, Garland A, et al. Effectiveness of brief training in cognitive behavioural therapy techniques for palliative care practitioners. Palliat Med 2006; 20: 579-584.

28 Coventry P, Genmmell I, Todd C. Psychological risk factors for hospital readmission in COPD patients on early discharge services: a cohort study. BMC Pulm Med 2011; 11: 49.

29 Puhan MA, Frey M, Buchi S, et al. The minimal important difference of the Hospital Anxiety and Depression Scale in patients with chronic obstructive pulmonary disease. Health Qual Life Outcomes 2008; 6: 46.

30 Jones PW, Harding G, Berry P, et al. Development and first validation of the COPD Assessment Test. Eur Respir J 2009; 34: 648-654

31 Szende A, Williams A, eds. Measuring Self-Reported Population Health: An International Perspective based on EQ-5D. EuroQol Group, 2004. https://euroqol.org/wp-content/uploads/2016/10/Measuring_Self-Reported_ Population_Health_-_An_International_Perspective_based_on_EQ-5D.pdf

32 Kon SC, Canavan JL, Jones SE, et al. Minimum clinically important difference for the COPD Assessment Test: a prospective analysis. Lancet Respir Med 2014; 2: 195-203. 
33 Picard S, Wilke C, Jung E, et al. Use of a preference-based measure of health (EQ-5D) in COPD and Asthma Respir Med 2007; 102: 319-336.

34 Bland M. An Introduction to Medical Statistics. 4th Edn. Oxford, Oxford University Press, 2015.

35 Rice WR. Analyzing tables of statistical tests. Evolution 1989; 43: 223-225.

36 Ramsey S, Willke R, Briggs A, et al. Good research practices for cost-effectiveness analysis alongside clinical trials: the ISPOR RCT-CEA Task Force report. Value Health 2005; 8: 521-533.

37 Manca A, Hawkins N, Sculpher MJ. Estimating mean QALYs in trial-based cost-effectiveness analysis: the importance of controlling for baseline utility. Health Econ 2005; 14: 487-496.

38 National Institute for Health and Clinical Excellence. Guide to the Methods of Technology Appraisal. Process and methods [PMG9]. National Institute for Health and Clinical Excellence, 2013.

39 Curtis L, Burns A. Unit Costs of Health and Social Care. Personal Social Services Research Unit. Canterbury, University of Kent, 2015.

40 Department of Health. Reference Costs. 2015-2016. https://assets.publishing.service.gov.uk/government/uploads/ system/uploads/attachment_data/file/577083/Reference_Costs_2015-16.pdf

41 Michie S, Abraham C, Whittington C, et al. Effective techniques in health eating and physical activity interventions: a meta-regression. Health Psychol 2014; 28: 690-701

42 McCabe C, Claxton K, Culyer A. The NICE cost effectiveness threshold: what it is and what that means. Pharmacoeconomics 2008; 26: 733-744.

43 Kunik ME, Roundy K, Veazey C. Surprisingly high prevalence of anxiety and depression in chronic breathing disorders. Chest 2005; 127: 120501211. 